\title{
Liraglutide and Insulin are Associated with a Decreased Risk of Acute Myocardial Infarction in Type 2 Diabetes Mellitus Patients
}

Jakob Starup-Linde ${ }^{1,2^{*}}$, Jan Scheel-Thomsen ${ }^{3}$, Michael Gejl ${ }^{2,4}$, Peter Vestergaard ${ }^{2,5}$, and Soeren Gregersen ${ }^{2}$

${ }^{1}$ Clinical Institute, Aalborg University, Denmark

${ }^{2}$ Department of Endocrinology and Internal Medicine (MEA), Aarhus University Hospital, Denmark

${ }^{3}$ Department of Neurology, Aalborg University Hospital, Denmark

${ }^{4}$ Department of Biomedicine, Aarhus University, Denmark

${ }^{5}$ Department of Endocrinology, Aalborg University Hospital, Denmark

*Corresponding author: Jakob Starup-Linde, Department of Endocrinology and Internal Medicine (MEA), Aarhus University Hospital, Tage-Hansens Gade 2, 8000 Aarhus C, Denmark, Tel: 0045 78467682; Fax: 0045 78467684; E-mail: jakolind@rm.dl

Rec date: May 07, 2014, Acc date: June 01, 2014, Pub date: June 07, 2014

Copyright: (c) 2014 Linde JS, et al. This is an open-access article distributed under the terms of the Creative Commons Attribution License, which permits unrestricted use, distribution, and reproduction in any medium, provided the original author and source are credited.

\begin{abstract}
Objective: Type 2 Diabetes Mellitus (DM) is associated with an increased risk of cardiovascular events. Glycemic control is beneficial to reduce acute myocardial infarction (AMI), however little is known whether a specific antidiabetic treatment is superior to another. This study aims at examining antidiabetic drugs and their association with a subsequent $\mathrm{AMI}$ in patients with DM.

Methods: A nested case-control study was conducted. Cases were patients with DM who subsequently suffered from AMI; controls were DM patients with no subsequent AMI after DM diagnosis. Using the Danish National Hospital Discharge Register, we included DM patients with information on date of DM diagnosis, date of AMI, and comorbidities. From the Central Region of Jutland, Denmark, medication use and biochemical parameters were
\end{abstract} collected. Multivariate logistic regression analyses were conducted.

Results: 10,727 DM patients were included. In multivariate analysis liraglutide $(\mathrm{OR}=0.386,95 \% \mathrm{Cl} 0.218-0.682)$ decreased the risk of AMI. An AMI prior to DM diagnosis (OR=36.004, 95\% Cl: 24.441-53.038), increasing age $(1.027,95 \% \mathrm{Cl} 1.016-1.039)$, increasing diabetes duration $(1.060,95 \% \mathrm{Cl} 1.043-1.077)$, male gender $(\mathrm{OR}=1.310$, $95 \% \mathrm{Cl} 1.025-1.676)$, a diagnosis of heart failure ( $\mathrm{OR}=2.083,95 \% \mathrm{Cl}: 1.459-2.973)$, and peripheral artery disease $(\mathrm{OR}=1.506,95 \% \mathrm{Cl}: 1.032-2.198)$ were all significantly associated with the risk of subsequent AMI. Neither insulin, $\beta-$ cell stimulans, biguanides, antihypertensive treatment nor usage of statins or antiarrhythmic drugs showed any significance.

When additional adjustment by biochemical risk markers was done insulin was significantly associated with a reduced risk of $\mathrm{AMI}(\mathrm{OR}=0.235,95 \% \mathrm{Cl} 0.073-0.757)$. .Furthermore, liraglutide and biguanides both showed a significant dose- and exposure time effects on OR for AMI.

Conclusion: We have found a strong association between the use of liraglutide and insulin, and a reduced risk of AMI in patients with DM when taking general risk factors into account.

Keywords: Type 2 Diabetes Mellitus; Acute Myocardial Infarction; Liraglutide; Biguanides; Insulin; $\beta$-cell stimulating drugs; Nested casecontrol study

\section{Abbreviations:}

CI: Confidence Interval; AMI: Acute Myocardial Infarction; ADA: American Diabetes Association; AHA: American Heart Association; CVD: Cardiovascular Disease; DM: Diabetes Mellitus: DDP4: Dipeptidyl Peptidase 4 (DDP4); GLP-1: Glucagon-like Peptide-1; OR: Odds Ratio

\section{Introduction}

The strong correlation between type 2 diabetes mellitus (DM) and cardiovascular morbidity and mortality is well established [1]. Patients with DM are characterized by serious micro- and macrovascular complications; the latter accounting for $52 \%$ of all diabetes fatalities [2] primarily due to stroke, myocardial infarction, and ischemic heart failure [3]. Combined guidelines from the American Heart Association (AHA) and the American Diabetes Association (ADA) suggests, in addition to lifestyle changes, to control risk factors by medical treatment of dyslipidemia, hypertension, coagulation and hyperglycemia as primary prevention of cardiovascular diseases in patients with DM [4]. ADA recommends a HbAlc level $<7.0 \%$ (53 $\mathrm{mmol} / \mathrm{mol}$ ) in general, but target levels as low as a normal HbAlc without causing hypoglycemia [4]. A meta-analysis of five randomized controlled trials found a beneficial effect of intensive glycemic control and concomitant lower $\mathrm{HbAlc}$ on cardiovascular disease (CVD), with a pooled reduction of $17 \%$ on myocardial infarction among DM patients [5]. However, it remains unknown whether a specific 
Citation: Starup-Linde J, Scheel-Thomsen J, Gejl M, Vestergaard P, Gregersen S (2014) Liraglutide and Insulin are Associated with a Decreased Risk of Acute Myocardial Infarction in Type 2 Diabetes Mellitus Patients. J Diabetes Metab 5: 389. doi: $10.4172 / 2155-6156.1000389$

Page 2 of 8

antidiabetic therapy is superior to the others in reducing myocardial infarction.

The Glucagon-Like Peptide-1 (GLP-1) receptor agonists are well validated antidiabetic medications. They mimic a range of physiological actions of native GLP-1 on glucose metabolism including glucose-dependent stimulation of insulin secretion and inhibition of glucagon secretion [6]. Native GLP-1 and GLP-1 analogues are reported to have several significant cardiovascular effects with the potential to reduce the excess mortality and morbidity associated with DM as well as in patients with cardiac disease and no diabetes [7-9]. However, evidence is conflicting and an animal study has shown no beneficial effect of liraglutide on myocardial infarct size [10]. The UK Prospective Diabetes Study (UKPDS) [11] reported a 33 $\%$ risk reduction of myocardial infarction in DM intensively treated with metformin. The reported effect may solely be due to improved glycemic control [11]. Two randomized clinical trials on DPP-4 inhibitors, designed to evaluate cardiac safety, reported largely neutral outcomes $[12,13]$. Both animal and human models confirm a GLP-1 mediated effect on the cardiovascular system, thus GLP-1 may in addition to improved glycemic control also have protective effects on cardiovascular disease. The aim of this study is to investigate whether a specific antidiabetic therapy is superior to other antidiabetic therapies in prevention of the first Acute Myocardial Infarction (AMI) in DM patients.

\section{Methods}

The present study was conducted as a nested case-control study in a cohort of DM patients. Causal effect is the parameter of interest; therefore an unmatched design was selected [14]. Using the Danish National Hospital Discharge Register we initially included all patients diagnosed with DM in the period 1977-2011. The registry covers all inpatient contacts from 1977 until 1994, afterwards also outpatient visits to hospitals, clinics and emergency rooms. Approval has been obtained by the Danish Data Protection Agency.

Cases were patients with DM who subsequently suffered from AMI; controls were DM patients with no occurrence of AMI at a later date than their DM diagnosis.

Table 1 presents the evaluated exposure variables along with their respective ICD-codes or ATC-codes.

Subjects with DM were extracted using ICD10 (E11) and ICD8 (250) codes. We only included patients diagnosed with DM. We rejected patients with 1) an unspecified DM diagnosis, 2) type $1 \mathrm{DM}$ diagnosis, 3) both a type 1 diabetes and a DM diagnosis, and 4) no information on date of diagnosis.

Cases were identified using the corresponding ICD10 (DI21-DI23) and ICD8 $(41009,41099)$ codes for AMI. A case was defined by an event subsequent to DM diagnosis. Only patients with a diagnosis of AMI at a later date than the DM diagnosis and after the start of the prescription registry (see below) were included in the case population. Due to a unique personal identifier it is possible to link the diagnoses to the same individual.

Comorbidities were extracted from the registry using their corresponding ICD codes. A proxy variable was created for alcohol (alcohol-related diagnoses) and hypertension (the use of antihypertensive drugs). Age was defined as the age of the individual at January 1, 2008. All cases were subsequent to this date, thus medication use was first collected from this date. The duration of DM
(DM duration) was defined by the time from the date of DM diagnosis to the end of the prescription register, December 31, 2011.

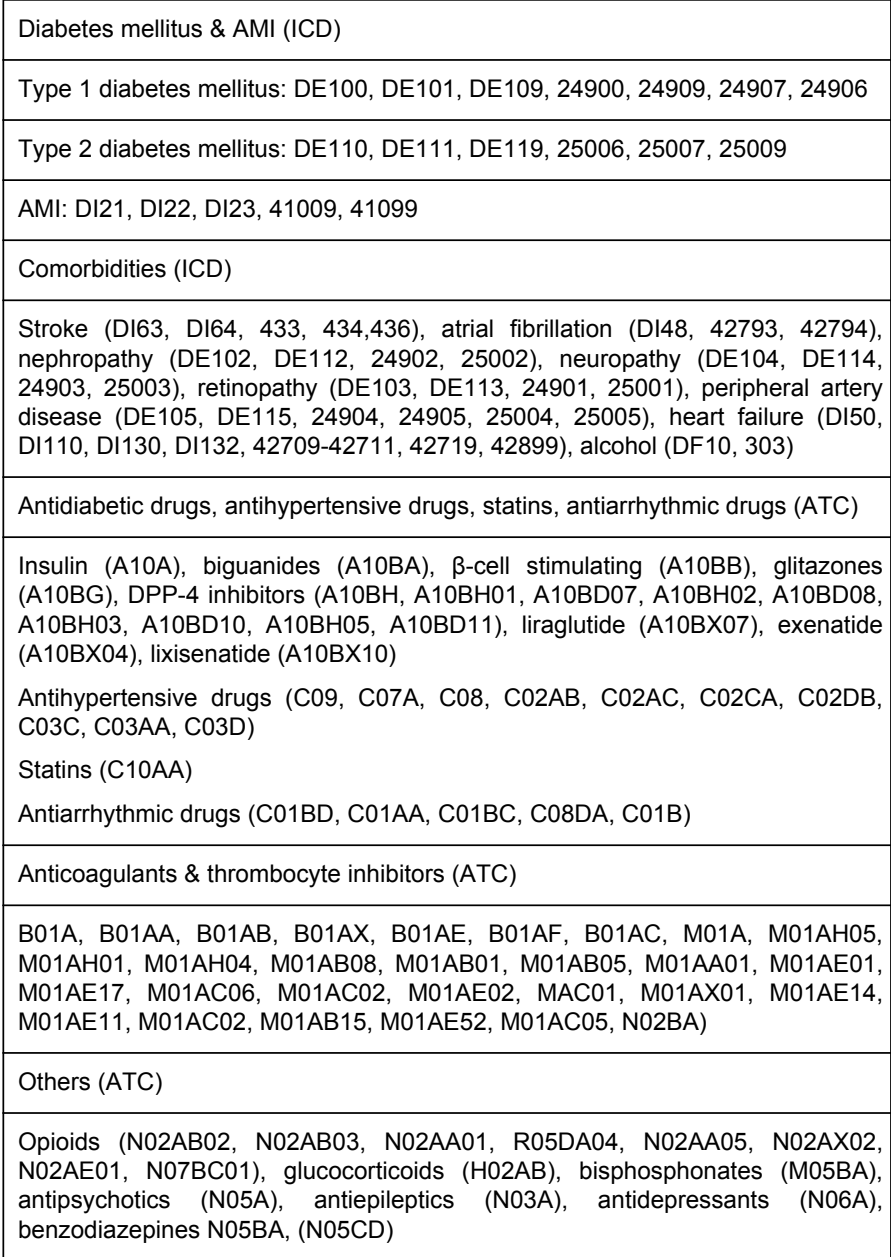

Table 1: Evaluated exposure variables used in the adjusted, covariate analysis ICD codes are shown as both ICD10 and ICD8

From the Central Region of Jutland, Denmark, medication use was collected from a registry. Any drugs bought are registered with ATC code, dose sold, and sales date for the period January 1, 2008, to December 31, 2011. Only prescription agents and not over-thecounter drugs are registered. As all sales are registered to the individual who redeemed the prescription, the capture and validity of data are high. Any medication sold is linked to a patient's unique personal identifier, and it is therefore possible to link prescriptions with diagnosis. We only included information on drugs prescribed before the date of AMI diagnosis. Only DM patients with information on medication use are included in the study. A total of 370 cases were available and 10,357 controls were available, all with information on pharmaceutical use. DM patients with no redeemed prescriptions on antidiabetic drugs (e.g. dietary treated) were also included in the analysis.

From the Central Region of Jutland, biochemistry (in the period 2008-2011) linked to the same unique personal identifier was extracted. All biochemical analyses were performed in an ISO standardized laboratory. Included biochemical analyses were restricted to the time before the date of AMI. 
Citation: Starup-Linde J, Scheel-Thomsen J, Gejl M, Vestergaard P, Gregersen S (2014) Liraglutide and Insulin are Associated with a Decreased Risk of Acute Myocardial Infarction in Type 2 Diabetes Mellitus Patients. J Diabetes Metab 5: 389. doi: $10.4172 / 2155-6156.1000389$

Page 3 of 8

Dose and treatment duration for liraglutide and biguanides were calculated. Duration was defined as the time between first redeemed prescription and the event of AMI (for cases) or before the end of follow up, December 31, 2011 (for controls). Dose was defined as the total daily dose redeemed before the event of AMI or before the end of follow up, December 31, 2011 (for controls). Both liraglutide duration and liraglutide dose were grouped by 25 th centile, 50th centile and 75 th centile. Biguanide duration was grouped by years of treatment and biguanide dose by daily dose intervals.

Statistical analysis was done using STATA 8. We used logistic regression to calculate unadjusted odds ratios (OR) and multivariate logistic regression to calculate the adjusted OR. In the following 'risk' is used synonymous for odds. This assumption can be made since less than $10 \%$ of the total analyzed population is cases.

We analyzed patients with diabetes, AMI, comorbidities and drug use. The DM patients received different combinations of treatment, and individual adjustment by usage of other pharmaceuticals including antidiabetics was performed in the model. In the multivariate analysis the following variables were included: previous AMI, age, DM duration, gender, atrial fibrillation, hypertension, alcohol related diagnosis, nephropathy, retinopathy, neuropathy, peripheral artery disease, usage of antiarrhythmic drugs, glitazones, vitamin $\mathrm{K}$ antagonists, heparin, pentasaccharides, argatroban, thrombocyte function inhibitors, acetylsalicylic acid, cyclooxygenase 2 inhibitors, nonselective cyclooxygenase inhibitors, buprenorphine, tramadol, oxycodone, morphine, codeine, fentanyl, pethidine, glucocorticoids, bisphosphonates, benzodiazepines, antipsychotics, antiepileptic drugs, statins, antidepressants, insulins, DPP-4 inhibitor, liraglutide, exenatide, biguanides, and $\beta$ cell stimulants.

A sensitivity analysis was conducted with the addition of biochemical variables to the model. This model also included HbAlc (\%), LDL cholesterol ( $\mathrm{mmol} / \mathrm{l})$, HDL cholesterol $(\mathrm{mmol} / \mathrm{l})$, total cholesterol ( $\mathrm{mmol} / \mathrm{l})$, triglycerides $(\mathrm{mmol} / \mathrm{l})$ and creatinine $(\mu \mathrm{mol} / \mathrm{l})$.

A secondary analysis was performed to compare mean biochemistry values (HbAlc, LDL, HDL, total cholesterol, triglycerides, creatinine and urinary albumin creatinine ratio) before and after liraglutide use. This analysis was conducted in all liraglutide users available. The analyses were performed in STATA 8 using paired t-test. Analysis on urinary albumin creatinine ratio was performed on log scale to achieve normal distribution.

\section{Results}

Table 2 presents the baseline characteristics of cases and controls. Among the population of DM patients in Denmark, 10,727 DM patients had information on comorbidities, and pharmaceutical use and were included in the study. Among the total population of 10,727 patients, mean age was $60.0 \pm 13.5$ years. Female-male ratio was 0.82 . Three percent (370) suffered from subsequent AMI. The mean duration of time from DM onset to subsequent AMI was $8.7 \pm 6.7$ years.

In the subgroup population, a total of 1,072 DM patients had information on biochemical risk markers and were included. Mean $( \pm \mathrm{SD})$ age was $63.1 \pm 12.2$ years. Female-male ratio was 1.01 . Four percent (44) suffered from subsequent AMI.

Cases had a mean age of 67.6 years, whereas controls were 59.7 years. Cases had longer mean diabetes duration of 10.5 years compared to 7.2 years in controls.

\begin{tabular}{|c|c|c|}
\hline & $\begin{array}{l}\text { Cases } \\
(n=370)\end{array}$ & $\begin{array}{l}\text { Controls } \\
(n=10,357)\end{array}$ \\
\hline Mean Age $\pm S D(y)$ & $67.6 \pm 12.2$ & $59.7 \pm 13.5$ \\
\hline Mean diabetes duration & $10.5 \pm 6.7$ & $7.2 \pm 6.4$ \\
\hline Males (n) & $241(65.1 \%)$ & $5,643(54.5 \%)$ \\
\hline Females (n) & $129(34.9 \%)$ & $4714(45.5 \%)$ \\
\hline Nephropathy (n) & $28(7.6 \%)$ & $541(5.2 \%)$ \\
\hline Neuropathy (n) & $19(5.1 \%)$ & $396(3.8 \%)$ \\
\hline Retinopathy (n) & $11(3.0 \%)$ & $215(2.1 \%)$ \\
\hline Peripheral artery disease & $44(11.9 \%)$ & $501(4.8 \%)$ \\
\hline Alcohol & $14(3.8 \%)$ & $366(3.5 \%)$ \\
\hline Previous AMI (n) & $80(21.6 \%)$ & $77(0.7 \%)$ \\
\hline Atrial fibrillation & $49(13.2 \%)$ & $848(8.2 \%)$ \\
\hline Insulin users(n) & $198(53.5 \%)$ & $4,547(43.9 \%)$ \\
\hline Biguanid users ( $n$ ) & $195(52.6 \%)$ & $7,646(73.8 \%)$ \\
\hline$\beta$-cell stimulating users $(n)$ & $137(37.0 \%)$ & $3,913(37.8 \%)$ \\
\hline Glitazones users ( $n$ ) & $2(0.5 \%)$ & $142(1.4 \%)$ \\
\hline DPP-4 inhibitors users (n) & $22(5.9 \%)$ & $1,159(11.2 \%)$ \\
\hline GLP-1: Liraglutide users ( $n$ ) & $14(3.8 \%)$ & $1,586(15.3 \%)$ \\
\hline GLP-1: Exenatide users ( $n$ ) & $10(2.7 \%)$ & $396(3.8 \%)$ \\
\hline Antiarrhythmic drugs & $44(11.9 \%)$ & $794(7.7 \%)$ \\
\hline Hypertension (n) & $328(88.6 \%)$ & $8,616(83.2 \%)$ \\
\hline Statins users $(n)$ & $279(75.4 \%)$ & $8,096(78.2 \%)$ \\
\hline
\end{tabular}

Table 2: Baseline characteristics of DM cases and controls $(n=10,727)$ used in the covariate adjusted analyses.

A small overweight of males were apparent among both cases and controls (64.5\% and $54.1 \%$, respectively). Nephropathy, retinopathy and neuropathy were rare in both cases and controls $(<10 \%)$. More cases had a previous AMI (21.6\%) and peripheral artery disease (11.9\%) compared to controls $(0.7 \%$ and $4.8 \%$, respectively). In both cases and controls more than $50 \%$ used biguanides and insulins, and $37 \%$ of cases and $38 \%$ of controls used $\beta$-cell stimulants, whereas only $3.8 \%$ of cases and $15.3 \%$ of controls used liraglutide. More than $75 \%$ of both cases and controls used statins and antihypertensives.

Table 3 presents unadjusted results for antidiabetic drugs, hypertension, and statins. In the raw analysis for the total study population both insulin and hypertension increase the risk of AMI, while biguanides, DPP-4 inhibitors, and liraglutide decrease the risk of AMI. Table 4 presents results from the adjusted analysis. In the multivariate analysis male gender, atrial fibrillation, nephropathy, retinopathy, neuropathy, former diagnosis of stroke, and an alcohol related diagnosis showed no significant effect on the risk of developing AMI. Increasing age (1.027, 95\%CI 1.016-1.039), increasing diabetes duration $(1.060,95 \% \mathrm{CI} 1.043-1.077)$, AMI prior to DM diagnosis $(\mathrm{OR}=36.004,95 \% \mathrm{CI}: 24.441-53.038)$, a diagnosis of heart failure $(\mathrm{OR}=$ 
Citation: Starup-Linde J, Scheel-Thomsen J, Gejl M, Vestergaard P, Gregersen S (2014) Liraglutide and Insulin are Associated with a Decreased Risk of Acute Myocardial Infarction in Type 2 Diabetes Mellitus Patients. J Diabetes Metab 5: 389. doi: $10.4172 / 2155-6156.1000389$

Page 4 of 8

2.083, 95\%CI: 1.459-2.973), and peripheral artery disease $(\mathrm{OR}=1.506$, 95\%CI: 1.032-2.198) all significantly increased the risk of subsequent AMI.

\begin{tabular}{|l|l|l|}
\hline & $\begin{array}{l}\text { Without biochemistry } \\
(\mathbf{n}=10,727)\end{array}$ & $\begin{array}{l}\text { With biochemistry } \\
(\mathbf{n = 1}, \mathbf{0 7 2})\end{array}$ \\
\hline Insulin & $1.471(1.195-1.811)$ & $1.564(0.855-2.861)$ \\
\hline Biguanide & $0.395(0.321-0.487)$ & $0.395(0.215-0.724)$ \\
\hline$\beta$-cell stimulating & $0.968(0.781-1.200)$ & $0.865(0.462-1.619)$ \\
\hline Glitazones & $0.391(0.096-1.585)$ & $-^{*}$ \\
\hline DPP-4 inhibitors & $0.502(0.325-0.775)$ & $0.636(0.194-2.090)$ \\
\hline GLP-1: Liraglutide & $0.217(0.127-0.372)$ & $-^{*}$ \\
\hline GLP-1: Exenatide & $0.699(0.370-1.320)$ & $1.571(0.203-12.165)$ \\
\hline Hypertension & $1.578(1.140-2.185)$ & $2.031(0.620-6.651)$ \\
\hline Statins & $0.856(0.673-1.090)$ & $1.025(0.449-2.339)$ \\
\hline
\end{tabular}

Table 3: Crude odds ratios (95\% CI) for the AMI outcome in DM patients Selected variables of pharmaceutical use are presented, $n=$ number, bold indicates significance ${ }^{\star}$ Predicts failure perfectly and not included in the analysis

\begin{tabular}{|c|c|c|c|c|c|c|}
\hline & \multicolumn{3}{|c|}{$\begin{array}{l}\text { Without biochemistry } \dagger \\
n=10,727\end{array}$} & \multicolumn{3}{|c|}{$\begin{array}{l}\text { With biochemistry† } \\
n=1,072\end{array}$} \\
\hline & $\mathrm{N}$ & $\begin{array}{l}\text { Odds } \\
\text { Ratio }\end{array}$ & $\begin{array}{l}\mathrm{P} \\
(95 \% \mathrm{Cl})\end{array}$ & $N$ & $\begin{array}{l}\text { Odds } \\
\text { Ratio }\end{array}$ & $\begin{array}{l}\mathrm{P} \\
(95 \% \mathrm{Cl})\end{array}$ \\
\hline Cases & $\begin{array}{l}370 \\
(3 \%)\end{array}$ & & & $\begin{array}{l}44 \\
(4 \% \\
)\end{array}$ & & \\
\hline \multicolumn{7}{|l|}{ Risk factors } \\
\hline Age & & 1.027 & $\begin{array}{l}0.001 \\
(1.016-1 . \\
039)\end{array}$ & & 1.049 & $\begin{array}{l}0.036 \\
(1.003-1.0 \\
98)\end{array}$ \\
\hline DM duration & & 1.060 & $\begin{array}{l}0.001 \\
(1.043-1 . \\
077)\end{array}$ & & 1.138 & $\begin{array}{l}0.001 \\
(1.071-1.2 \\
09)\end{array}$ \\
\hline AMI prior to DM & 157 & 36.004 & $\begin{array}{l}0.001 \\
(24.441-5 \\
3.038)\end{array}$ & 9 & $\begin{array}{l}498.28 \\
7\end{array}$ & $\begin{array}{l}0.001 \\
(34.367-72 \\
24.569)\end{array}$ \\
\hline Former stroke & 873 & 1.203 & $\begin{array}{l}0.273 \\
(0.864-1 . \\
675)\end{array}$ & 104 & 0.633 & $\begin{array}{l}0.462 \\
(0.187-2.1 \\
44)\end{array}$ \\
\hline Atrial fibrillation & 897 & 1.272 & $\begin{array}{l}0.278 \\
(0.823-1 . \\
966)\end{array}$ & 117 & 1.039 & $\begin{array}{l}0.959 \\
(0.238-4.5 \\
37)\end{array}$ \\
\hline Nephropathy & 569 & 1.062 & $\begin{array}{l}0.784 \\
(0.690-1 . \\
636)\end{array}$ & 51 & 2.197 & $\begin{array}{l}0.251 \\
(0.573-8.4 \\
23)\end{array}$ \\
\hline Neuropathy & 415 & 1.079 & $\begin{array}{l}0.770 \\
(0.649-1 . \\
793)\end{array}$ & 34 & 2.799 & $\begin{array}{l}0.128 \\
(0.744-10 . \\
532)\end{array}$ \\
\hline
\end{tabular}

\begin{tabular}{|c|c|c|c|c|c|c|}
\hline Retinopathy & 226 & 0.894 & $\begin{array}{l}0.739 \\
(0.464-1 . \\
725)\end{array}$ & 26 & 0.259 & $\begin{array}{l}0.227 \\
(0.029-2.3 \\
19)\end{array}$ \\
\hline $\begin{array}{l}\text { Peripheral artery } \\
\text { disease }\end{array}$ & 545 & 1.506 & $\begin{array}{l}0.034 \\
(1.032-2 . \\
198)\end{array}$ & 56 & 2.086 & $\begin{array}{l}0.230 \\
(0.628-6.9 \\
24)\end{array}$ \\
\hline Heart failure & 582 & 2.083 & $\begin{array}{l}0.001 \\
(1.459-2 . \\
973)\end{array}$ & 70 & 2.289 & $\begin{array}{l}0.151 \\
(0.740-7.0 \\
84)\end{array}$ \\
\hline Male gender & 5,884 & 1.310 & $\begin{array}{l}0.031 \\
(1.025-1 . \\
676)\end{array}$ & 534 & 1.443 & $\begin{array}{l}0.417 \\
(0.596-3.4 \\
93)\end{array}$ \\
\hline Alcohol & 380 & 1.418 & $\begin{array}{l}0.238 \\
(0.794-2 . \\
533)\end{array}$ & 45 & 0.741 & $\begin{array}{l}0.807 \\
(0.067-8.2 \\
36)\end{array}$ \\
\hline \multicolumn{7}{|l|}{$\begin{array}{l}\text { Antidiabetic } \\
\text { drugs }\end{array}$} \\
\hline Insulin & 4,745 & 1.120 & $\begin{array}{l}0.400 \\
(0.860-1 . \\
459)\end{array}$ & 423 & 0.235 & $\begin{array}{l}0.015 \\
(0.073-0.7 \\
57)\end{array}$ \\
\hline Biguanides & 7,841 & 0.800 & $\begin{array}{l}0.087 \\
(0.619-1 . \\
033)\end{array}$ & 425 & 0.473 & $\begin{array}{l}0.112 \\
(0.188-1.1 \\
91)\end{array}$ \\
\hline$\beta$-cell stimulating & 4,050 & 1.130 & $\begin{array}{l}0.348 \\
(0.875-1 \\
458)\end{array}$ & 847 & 0.719 & $\begin{array}{l}0.490 \\
(0.286-1.8 \\
36)\end{array}$ \\
\hline Glitazones & 144 & 0.643 & $\begin{array}{l}0.541 \\
(0.156-2 \\
651)\end{array}$ & & -* & $-^{*}$ \\
\hline DPP-4 inhibitors & 1,181 & 0.816 & $\begin{array}{l}0.391 \\
(0.512-1 . \\
299)\end{array}$ & 109 & 0.914 & $\begin{array}{l}0.908 \\
(0.199-4.2 \\
00)\end{array}$ \\
\hline $\begin{array}{l}\text { GLP-1: } \\
\text { Liraglutide }\end{array}$ & 1,600 & 0.386 & $\begin{array}{l}0.001 \\
(0.218-0 . \\
682)\end{array}$ & 180 & -* $^{*}$ & $-^{*}$ \\
\hline $\begin{array}{l}\text { GLP-1: } \\
\text { Exenatide }\end{array}$ & 406 & 1.651 & $\begin{array}{l}0.154(0.8 \\
29-3.288)\end{array}$ & 16 & 2.096 & $\begin{array}{l}0.555 \\
(0.180-24 . \\
409)\end{array}$ \\
\hline \multicolumn{7}{|l|}{ Bloodpressure } \\
\hline Hypertension & 8,944 & 0.934 & $\begin{array}{l}0.735 \\
(0.631-1 . \\
384)\end{array}$ & 936 & 1.282 & $\begin{array}{l}0.751 \\
(0.276-5.9 \\
59)\end{array}$ \\
\hline \multicolumn{7}{|l|}{ Atrial fibrillation } \\
\hline $\begin{array}{l}\text { Antiarrhythmic } \\
\text { drugs }\end{array}$ & 838 & 0.968 & $\begin{array}{l}0.883 \\
(0.628-1 . \\
493)\end{array}$ & 98 & 0.718 & $\begin{array}{l}0.679 \\
(0.149-3.4 \\
51)\end{array}$ \\
\hline \multicolumn{7}{|l|}{ Cholesterol } \\
\hline Statins & 8,375 & 0.900 & $\begin{array}{l}0.474 \\
(0.674-1 . \\
201)\end{array}$ & 898 & 0.689 & $\begin{array}{l}0.503 \\
(0.232-2.0 \\
47)\end{array}$ \\
\hline
\end{tabular}

$\mathrm{DM}=$ diabetes mellitus; $\mathrm{n}=$ amount; $\mathrm{Cl}=$ confidence interval; Bold indicate significance

*Predicts failure perfectly and not included in the analysis

†Adjusted for: previous AMI, age, DM duration, gender, atrial fibrillation, hypertension, alcohol related diagnosis, nephropathy, retinopathy, neuropathy, peripheral artery disease, usage of antiarrhythmic drugs, glitazones, vitamin $\mathrm{K}$ 
Citation: Starup-Linde J, Scheel-Thomsen J, Gejl M, Vestergaard P, Gregersen S (2014) Liraglutide and Insulin are Associated with a Decreased Risk of Acute Myocardial Infarction in Type 2 Diabetes Mellitus Patients. J Diabetes Metab 5: 389. doi: $10.4172 / 2155-6156.1000389$

Page 5 of 8

antagonists, heparin, pentasaccharides, argatroban, thrombocyte function inhibitors, acetylsalicylic acid, cyclooxygenase 2 inhibitors, nonselective cyclooxygenase inhibitors, buprenorphine, tramadol, oxycodone, morphine, codeine, fentanyl, pethidine, glucocorticoids, bisphosphonates, benzodiazepines, antipsychotics, antiepileptic drugs, statins, antidepressants, insulins, DPP-4 inhibitor, liraglutide, exenatide, biguanides, and $\beta$ cell stimulants.

$\ddagger$ Adjusted for: LDL, HDL, total cholesterol, $\mathrm{HbA1c}$, creatinine and triglycerides

Table 4: Multivariate analysis DM patients Analyses performed with and without the adjustment of biochemical variables Odds ratio shows the risk of developing AMI if the case has received a pharmaceutical drug prior to AMI occurrence.

Neither hypertension nor usage of statins or antiarrhythmic drugs showed any significance.

Of the antidiabetic drugs, insulin (OR=1.120, 95\%CI: 0.860-1.459), biguanides $(\mathrm{OR}=0.800,95 \% \mathrm{CI} 0.619-1.033)$, and $\beta$-cell stimulating drugs $(\mathrm{OR}=1.130,95 \% \mathrm{CI} \quad 0.875-1.458)$ showed no statistical significance. Liraglutide $(\mathrm{OR}=0.386,95 \% \mathrm{CI} 0.218-0.682)$ reduced the risk of AMI significantly. Neither glitazones nor exenatide or DDP-4 inhibitors had a significant risk reducing or increasing effect. Results did not change in an analysis matched by age (by five year intervals), previous AMI, gender and diabetes duration (by two year intervals) (results not presented) with the exception of statins which was associated with a significant reduction of AMI $(\mathrm{P}=0.03)$ and a loss of statistical significance for peripheral artery disease.

In the sensitivity analysis adjusting for LDL, HDL, total cholesterol, triglycerides, $\mathrm{HbAlc}$ and creatinine, the risk increasing effect of prior AMI (OR=498.287, 95\%CI: 34.367-7224.569) remained significant, whereas insulin significantly reduced risk of AMI $(\mathrm{OR}=0.235,95 \% \mathrm{CI}$ 0.073-0.757). Other patient characteristics were without significance. Liraglutide was not included in this analysis since none of the 180 subjects receiving liraglutide had a subsequent AMI. Among these 180 liraglutide users the mean age was $55.1( \pm 10.1)$ years, mean DM duration was $7.9( \pm 5.9)$ years, $52 \%$ were male, $62 \%$ received insulin, $96 \%$ received biguanides, $54 \%$ received $\beta$-cell stimulants, $0 \%$ received glitazones, $91 \%$ received antihypertensive drugs, $94 \%$ received statins, and $63 \%$ received thrombocyte function inhibitors.

The results concerning the antidiabetic drugs are illustrated in Figure 1. No effect modification between use of both liraglutide and biguanide $(\mathrm{p}=0.89)$ or between liraglutide and insulin $(\mathrm{p}=0.446$ were present. Figure 2 presents results on the effect of liraglutide and biguanide exposure duration, as well as the effect of liraglutide and biguanides dose on the risk of developing AMI. It revealed that longer duration and increased dose of both treatments decreased the risk of AMI compared to non users. Additional results are provided in the Table 5 and 6.

Bold indicate significance a†Adjusted for: previous AMI, age, DM duration, gender, atrial fibrillation, hypertension, alcohol related diagnosis, nephropathy, retinopathy, neuropathy, peripheral artery disease, usage of antiarrhythmic drugs, glitazones, vitamin $\mathrm{K}$ antagonists, heparin, pentasaccharides, argatroban, thrombocyte function inhibitors, acetylsalicylic acid, cyclooxygenase 2 inhibitors, nonselective cyclooxygenase inhibitors, buprenorphine, tramadol, oxycodone, morphine, codeine, fentanyl, pethidine, glucocorticoids, bisphosphonates, benzodiazepines, antipsychotics, antiepileptic drugs, statins, antidepressants, insulins, DPP-4 inhibitor, liraglutide, exenatide, biguanides, and $\beta$ cell stimulants. ${ }^{*}$ Significantly different from nonusers $(\mathrm{p}<0.05)$.

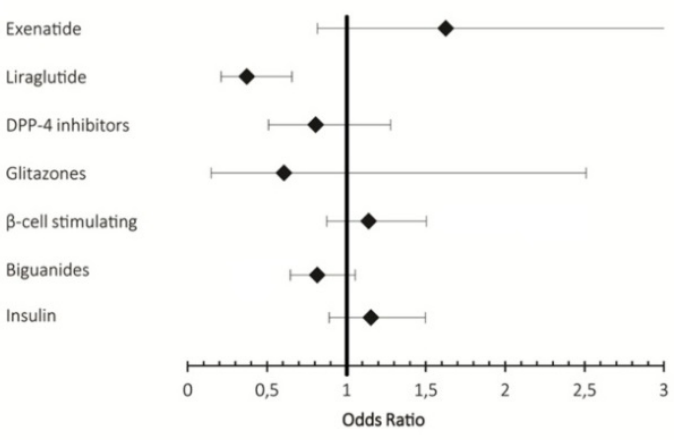

Figure 1: Effects of antidiabetic drugs and statins on risk of developing AMI in patients with DM. Results are unadjusted for biochemistry. Results are shown as odds ratio with 95\% error margins.

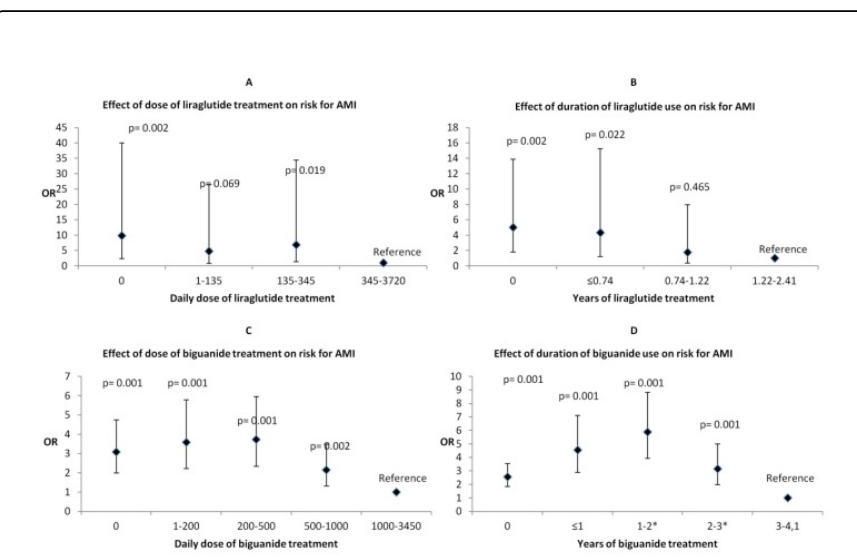

Figure 2: Effects of biguanide and liraglutide duration and dose on the risk of AMI. Results are unadjusted for biochemistry. A) Effect of liraglutide dose B) Effect of liraglutide duration C) Effect of biguanide dose D) Effect of biguanide duration

As outlined in the tables, results are also adjusted for a number of other pharmaceuticals, e.g. anticoagulants and thrombocyte inhibitors; however their effects are not presented here due to the scope of the study.

Secondary analyses involving all DM patients revealed that liraglutide treatment significantly decreased HbAlc (mean $0.47 \%$, SD $0.01, \mathrm{P}<0.0001$ ), LDL cholesterol (mean $0.14 \mathrm{mmol} / \mathrm{l}$, SD 0.54, $\mathrm{P}=0.0004)$, total cholesterol $(0.23 \mathrm{mmol} / \mathrm{l}, \mathrm{SD} 0.67, \mathrm{P}<0.0001)$, and triglycerides (mean $0.26 \mathrm{mmol} / \mathrm{l}, \mathrm{SD} 1.09, \mathrm{P}=0.0230$ ). HDL cholesterol (mean $0.20 \mathrm{mmol} / \mathrm{l}, \mathrm{SD} 0.15, \mathrm{P}=0.08$ ) did not change significantly, and 
Citation: Starup-Linde J, Scheel-Thomsen J, Gejl M, Vestergaard P, Gregersen S (2014) Liraglutide and Insulin are Associated with a Decreased Risk of Acute Myocardial Infarction in Type 2 Diabetes Mellitus Patients. J Diabetes Metab 5: 389. doi: $10.4172 / 2155-6156.1000389$

Page 6 of 8

creatinine (mean $3.35 \mu \mathrm{mol} / \mathrm{l}, \mathrm{SD} 10.24, \mathrm{P}<0.0001$ ) significantly increased. Log Urinary albumin creatinine ratio decreased after liraglutide exposure, though not statistically significant $(0.13 \mathrm{mg} / \mathrm{g}, \mathrm{SD}$ $1.09, \mathrm{P}=0.27)$.

\begin{tabular}{|l|l|l|l|}
\hline & \multicolumn{3}{|l|}{ Liraglutide duration and dosea $\mathbf{n = 1 0 , 7 2 7}$} \\
\hline & $\mathrm{N}$ & Odds Ratio & $\mathrm{P}(95 \% \mathrm{Cl})$ \\
\hline Duration (years) & & & \\
\hline 0 & 9,127 & 5.024 & $0.002(1.816-13.898)$ \\
\hline $0-0.74$ & 397 & 4.336 & $0.022(1.232-15.259)$ \\
\hline $0.74-1.22^{*}$ & 394 & 1.756 & $0.465(0.387-7.963)$ \\
\hline $1.22-2.41^{*}$ & 809 & 1 ref & \\
\hline
\end{tabular}

Dose (sum daily doses of $1.2 \mathrm{mg}$ )

\begin{tabular}{|l|l|l|l|}
\hline 0 & 9,183 & 9.522 & $0.002(2.332-38.885)$ \\
\hline $1-135$ & 345 & 4.915 & $0.069(0.883-27.355)$ \\
\hline $135-345$ & 380 & 6.879 & $0.019(1.366-34.656)$ \\
\hline $345-3,720^{*}$ & 819 & 1 ref & \\
\hline
\end{tabular}

Table 5: Effects of liraglutide duration and dose in DM patients Results are unadjusted for biochemistry. Odds ratio shows the risk of developing AMI if the case has received certain duration and certain total daily dose of liraglutide.

\begin{tabular}{|l|l|l|l|}
\hline & \multicolumn{3}{|l|}{ Biguanides duration and dose ${ }^{\mathrm{a}} \mathbf{n}=10,727$} \\
\hline & $\mathrm{N}$ & Odds Ratio & $\mathrm{P}(95 \% \mathrm{Cl})$ \\
\hline $\begin{array}{l}\text { Duration } \\
\text { (years) }\end{array}$ & & & \\
\hline 0 & 2,886 & 2.560 & $0.001(1.843-3.556)$ \\
\hline $0-1$ & 850 & 4.544 & $0.001(2.901-7.120)$ \\
\hline $1-2$ & 1,004 & 5.890 & $0.001(3.932-8.824)$ \\
\hline $2-3$ & 809 & 3.161 & $0.001(1.996-5.005)$ \\
\hline $3-4.1^{*}$ & 5,007 & 1 ref & \\
\hline
\end{tabular}

Dose (sum daily doses of $2 \mathrm{~g}$ metformin )

\begin{tabular}{|l|l|l|l|}
\hline 0 & 2,896 & 3.080 & $0.001(1.996-4.753)$ \\
\hline $1-200$ & 1,530 & 3.595 & $0.001(2.231-5.791)$ \\
\hline $200-500$ & 1,552 & 3.736 & $0.001(2.340-5.964)$ \\
\hline $500-1000^{*}$ & 1,916 & 2.150 & $0.002(1.323-3.495)$ \\
\hline $1000-3450^{*}$ & 2,833 & 1 ref & \\
\hline
\end{tabular}

Table 6: Effects of biguanide duration and dose in DM patients Results are unadjusted for biochemistry. Odds ratio shows the risk of developing AMI if the case has received certain duration and certain total daily dose of biguanides.

\section{Discussion}

Our results show that in a real world cohort of patients with DM, liraglutide decreased the risk of AMI. Liraglutide showed an apparent $62.8 \%$ risk reduction of AMI. When results were adjusted for biochemical risk markers, insulin statistically significantly decreased the risk of AMI. Although biguanides did not influence AMI risk; a clear dose and duration response were apparent towards beneficial effect of increasing dose and increasing duration.

Statins surprisingly did not influence the risk of AMI [15], however this may be due to the fact that most patients received statin treatment (78 \%) and the population is combined of DM patients with both elevated and normal cholesterol levels, thus those not receiving the treatment may have habitual normal cholesterol levels.

The effect of insulin was tied to biochemical markers and may reflect a more severe disease in individuals treated with insulin. The opposite directions of the insulin estimates among DM patients (dependent of the adjustment of biochemical risk markers) may be due to a more severe disease, which was reflected in the biochemical risk markers. It was not possible to evaluate the effect of liraglutide, due to no cases in the group with information on biochemistry. The 180 liraglutide users not included in the analyses adjusted for biochemical markers were younger; however, based on their use of multiple antidiabetic drugs simultaneously, they were not a more healthy subgroup. This supports a beneficial effect of liraglutide. The observed association between liraglutide and a decreased risk of AMI is further supported by our finding of a dose- and duration-response trend. The effects of liraglutide on biochemical markers were assessed as a secondary analysis, to determine whether reduction in lipids or HbAlc may influence the findings. Beneficial effects of liraglutide were found on HbA1c, LDL, total cholesterol and triglycerides after liraglutide exposure. Such pleiotropic effects on cholesterol have previously been revealed by Vilsboll et al. [16]. A decrease in cholesterol and HbA1c may explain part of the reduced risk of AMI among liraglutide users.

Several factors may explain the observed reduction in AMI events among liraglutide users, e.g. improvements in CVD risk factors, including weight, glucose, lipids status, and blood pressure [17]. Furthermore, clinical trials of native GLP-1 and agonists suggest reduced atherosclerosis development and improved endothelial function [18], increased myocardial blood flow, improved myocardial glucose metabolism in the most insulin resistant subjects $[19,20]$, reduction of oxidative stress, and attenuation of proinflammatory cell activation [21]. But despite many proposed mechanisms the reasons for apparent lower risk of developing CVD are still poorly understood. GLP-1 and analogues exerts their actions through the GLP-1 receptor. The GLP-1 receptor may be present in cells of many tissues including the vascular endothelium, cardiomyocytes, endocardium, and smooth muscle cells. Some cardiac effects of GLP-1R agonists may be exerted via other receptors [22]. Prior studies have demonstrated the presence of GLP-1 receptors in the myocardium [22,23], but recent papers [24-26] have questioned the interpretation of data obtained using commercially available GLP-1 antisera to detect the authentic GLP-1 receptor.

Observational studies have linked hyperglycemia and CVD, but trials have not consistently confirmed that intensified glycemic control reduces CVD [27-29]; in fact an increased mortality in the ACCORD trial was associated with hypoglycemia [30]. GLP-1 analogues and biguanides are considered as agents with low risk of hypoglycaemia $[31,32]$. 
Citation: Starup-Linde J, Scheel-Thomsen J, Gejl M, Vestergaard P, Gregersen S (2014) Liraglutide and Insulin are Associated with a Decreased Risk of Acute Myocardial Infarction in Type 2 Diabetes Mellitus Patients. J Diabetes Metab 5: 389. doi: $10.4172 / 2155-6156.1000389$

Page 7 of 8

In contrast to our results, the most comprehensive meta-analysis of the GLP-1 analogues did not find any significant GLP-1 effect on CVD events as a whole [33], but this meta-analysis did not distinguish between the CVDs. However, our results agree with the large randomized trials conducted on DPP-4 inhibitors, as we also found a neutral effect $[12,13]$.

The study by Best et al. [34] based on the Life Link database found a protective effect of exenatide use on cardiovascular events compared to all other antidiabetics in DM patients. We could not confirm these findings; several significant differences between [34] and our study are present however. In this large study [34] 21,764 received exenatide treatment, thus the lack of effect in our study may be due to power, as only 406 received exenatide treatment [34]. Furthermore, all other antidiabetics than exenatide [34] were grouped as one, whereas the antidiabetics were handled individually in our study. The patients were all insured and may represent a selected group of patients [34], whereas the Danish registries cover the entire national population. The differences between the effects of exenatide and liraglutide in our study may be due to either power as a smaller number of individuals received exenatide, or due to differences in molecular structure and differences in resemblance of native GLP-1. Further investigations should explore the effects of exenatide and liraglutide and determine whether any differences are present.

Our results support previous findings of a beneficial effect of insulin on myocardial infarction in UKPDS [11]. In contrast to our findings, intensive biguanide and sulphonylurea therapy decreased risk of myocardial infarction by $15 \%$ among DM patients in the UKPDS [11], however the users were compared with patients on dietary restriction and no other therapies.

The study is limited by its retrospective design and thus causality can only be speculative. In addition, important information on lifestyle risk factors such as physical activity, smoking, alcohol, diet, and weight were not available and may restrict our results. It is not possible, based on the registries, to determine whether the liraglutide users were healthier than non-users. Pottegard et al. [35] showed that treatment with liraglutide was not associated with any changes in concomitant treatment with antihypertensives, cholesterol lowering drugs or anticoagulants and $38 \%$ of liraglutide users also used insulin. Thus, patients treated with liraglutide seem no different compared to patients treated with other antidiabetic drugs.

Furthermore, liraglutide was first registered in Denmark in 2009, which may influence the results; however in an analysis limited to cases subsequent to the release of liraglutide, liraglutide still significantly decreased the risk of AMI (results not presented in the paper). The prescription registry provides information on pharmaceuticals bought and not solely on drugs actually used; however, due to the necessity of the antidiabetic therapy, and the fact that individuals are inclined to use bought drugs, we assume this is only a minor restriction to the results. Patients may have switched antidiabetic therapy during the study period and will therefore be registered as a user of multiple drugs. To comply with this possible limitation adjustments have been made by individual antidiabetic drugs (e.g. each antidiabetic drug was a separate variable in the multivariate analyses).

Our results, in a real world cohort, showed beneficial effects of insulin and liraglutide of the antidiabetic therapies when adjusted by each other and other antidiabetic therapies. We are, to our knowledge, the first to report an AMI reducing effect of insulin when adjusted by HbA1c, total cholesterol, LDL, HDL, triglycerides and creatinine.

In conclusion, we have found a strong association between the use of liraglutide and insulin, and a reduced risk of AMI in patients with DM when taking general risk factors into account. The association of liraglutide was dose and duration dependent and highlights the health benefits of the drug. To establish whether liraglutide and insulin are superior to other antidiabetic drugs in AMI risk reduction, randomized clinical trials are needed. Currently the results from the Liraglutide Effect and Action in Diabetes: Evaluation of cardiovascular outcome Results (LEADER) [36] trial is awaited.

\section{Contribution Statement}

S.G., J.S-L and P.V. planned the study. J.S-L. and P.V. researched data and conducted the statistics. J.S-T., J.S-L, M.G., S.G. and P.V. wrote and reviewed the manuscript. P.V and S.G. are the guarantors of this work and, as such, had full access to all the data in the study and takes responsibility for the integrity of the data and the accuracy of the data analysis.

\section{Acknowledgement}

An unrestricted grant was received from Novo Nordisk A/S to support the study. Novo Nordisk A/S did not read, comment or correct the manuscript before submission.

\section{References}

1. Schernthaner G (1996) Cardiovascular mortality and morbidity in type-2 diabetes mellitus. Diabetes Res Clin Pract 31 Suppl: S3-13.

2. Morrish NJ, Wang SL, Stevens LK, Fuller JH, Keen H (2001) Mortality and causes of death in the WHO Multinational Study of Vascular Disease in Diabetes. Diabetologia 44 Suppl 2: S14-21.

3. Gu K, Cowie CC, Harris MI (1999) Diabetes and decline in heart disease mortality in US adults. JAMA 281: 1291-1297.

4. Buse JB, Ginsberg HN, Bakris GL, Nathaniel G. Clark, Fernando Costa et al. (2007) Primary prevention of cardiovascular diseases in people with diabetes mellitus: a scientific statement from the American Heart Association and the American Diabetes Association. Circulation 115:114-126

5. Ray KK, Seshasai SR, Wijesuriya S, Sivakumaran R, Nethercott S, et al. (2009) Effect of intensive control of glucose on cardiovascular outcomes and death in patients with diabetes mellitus: a meta-analysis of randomised controlled trials. Lancet 373: 1765-1772.

6. Ravassa S, Zudaire A, Díez J (2012) GLP-1 and cardioprotection: from bench to bedside. Cardiovasc Res 94: 316-323.

7. Lønborg J, Vejlstrup N, Kelbæk H, Bøtker HE, Kim WY, et al. (2012) Exenatide reduces reperfusion injury in patients with ST-segment elevation myocardial infarction. Eur Heart J 33: 1491-1499.

8. Nikolaidis LA, Mankad S, Sokos GG, Miske G, Shah A, et al. (2004) Effects of glucagon-like peptide-1 in patients with acute myocardial infarction and left ventricular dysfunction after successful reperfusion. Circulation 109: 962-965.

9. Sokos GG, Nikolaidis LA, Mankad S, Elahi D, Shannon RP (2006) Glucagon-like peptide-1 infusion improves left ventricular ejection fraction and functional status in patients with chronic heart failure. J Card Fail 12: 694-699.

10. Kristensen J, Mortensen UM, Schmidt M, Nielsen PH, Nielsen TT, et al. (2009) Lack of cardioprotection from subcutaneously and preischemic administered liraglutide in a closed chest porcine ischemia reperfusion model. BMC Cardiovasc Disord 9: 31. 
Citation: Starup-Linde J, Scheel-Thomsen J, Gejl M, Vestergaard P, Gregersen S (2014) Liraglutide and Insulin are Associated with a Decreased Risk of Acute Myocardial Infarction in Type 2 Diabetes Mellitus Patients. J Diabetes Metab 5: 389. doi: $10.4172 / 2155-6156.1000389$

Page 8 of 8

11. Holman RR, Paul SK, Bethel MA, Matthews DR, Neil HA (2008) 10-year follow-up of intensive glucose control in type 2 diabetes. $\mathrm{N}$ Engl J Med 359: 1577-1589.

12. Scirica BM, Bhatt DL, Braunwald E, Steg PG, Davidson J, et al. (2013) Saxagliptin and cardiovascular outcomes in patients with type 2 diabetes mellitus. N Engl J Med 369: 1317-1326.

13. White WB, Cannon CP, Heller SR, Nissen SE, Bergenstal RM, et al (2013) Alogliptin after acute coronary syndrome in patients with type 2 diabetes. N Engl J Med 369: 1327-1335.

14. Rose S, Laan MJ (2009) Why match? Investigating matched case-control study designs with causal effect estimation. Int J Biostat 5: Article 1.

15. Colhoun HM, Betteridge DJ, Durrington PN, Hitman GA, Neil HA, et al. (2004) Primary prevention of cardiovascular disease with atorvastatin in type 2 diabetes in the Collaborative Atorvastatin Diabetes Study (CARDS): multicentre randomised placebo-controlled trial. Lancet 364:685-696

16. Vilsbøll T, Christensen M, Junker AE, Knop FK, Gluud LL (2012) Effects of glucagon-like peptide-1 receptor agonists on weight loss: systematic review and meta-analyses of randomised controlled trials. BMJ 344: d7771.

17. Mundil D, Cameron-Vendrig A, Husain M (2012) GLP-1 receptor agonists: a clinical perspective on cardiovascular effects. Diab Vasc Dis Res 9: 95-108.

18. Sivertsen J, Rosenmeier J, Holst JJ, Vilsbøll T (2012) The effect of glucagon-like peptide 1 on cardiovascular risk. Nat Rev Cardiol 9: 209-222.

19. Gejl M, Søndergaard HM, Stecher C, Bibby BM, Møller N, et al. (2012) Exenatide alters myocardial glucose transport and uptake depending on insulin resistance and increases myocardial blood flow in patients with type 2 diabetes. J Clin Endocrinol Metab 97: E1165-9

20. Gejl M, Lerche S1, Mengel A2, Møller N2, Bibby BM3, et al. (2014) Influence of GLP-1 on myocardial glucose metabolism in healthy men during normo- or hypoglycemia. PLoS One 9: e83758.

21. Chinda K, Chattipakorn S, Chattipakorn N (2012) Cardioprotective effects of incretin during ischaemia-reperfusion. Diab Vasc Dis Res 9: 256-269.

22. Ban K, Noyan-Ashraf MH, Hoefer J, Bolz SS, Drucker DJ, et al. (2008) Cardioprotective and vasodilatory actions of glucagon-like peptide 1 receptor are mediated through both glucagon-like peptide 1 receptordependent and -independent pathways. Circulation 117: 2340-2350.

23. Bhashyam S, Fields AV, Patterson B, Testani JM, Chen L et al. (2010) Glucagon-like peptide-1 increases myocardial glucose uptake via p38alpha MAP kinase-mediated, nitric oxide-dependent mechanisms in conscious dogs with dilated cardiomyopathy. Circ Heart Fail 3: 512-521
24. Panjwani N, Mulvihill EE, Longuet C, Yusta B, Campbell JE, et al. (2013) GLP-1 receptor activation indirectly reduces hepatic lipid accumulation but does not attenuate development of atherosclerosis in diabetic male ApoE(-/-) mice. Endocrinology 154: 127-139.

25. Kim M, Platt MJ, Shibasaki T, Quaggin SE, Backx PH, et al. (2013) GLP-1 receptor activation and Epac2 link atrial natriuretic peptide secretion to control of blood pressure. Nat Med 19: 567-575.

26. Pyke C, Knudsen LB (2013) The glucagon-like peptide-1 receptor--or not? Endocrinology 154: 4-8

27. Duckworth W, Abraira C, Moritz T, Reda D, Emanuele N, et al. (2009) Glucose control and vascular complications in veterans with type 2 diabetes. N Engl J Med 360: 129-139.

28. Action to Control Cardiovascular Risk in Diabetes Study Group, Gerstein HC, Miller ME, Byington RP, Goff DC Jr, et al. (2008) Effects of intensive glucose lowering in type 2 diabetes. N Engl J Med 358: 2545-2559.

29. ADVANCE Collaborative Group, Patel A, MacMahon S, Chalmers J, Neal B, et al. (2008) Intensive blood glucose control and vascular outcomes in patients with type 2 diabetes. N Engl J Med 358: 2560-2572.

30. Bonds DE, Miller ME, Bergenstal RM, Buse JB, Byington RP, et al. (2010) The association between symptomatic, severe hypoglycaemia and mortality in type 2 diabetes: retrospective epidemiological analysis of the ACCORD study. BMJ 340: b4909.

31. Rojas LB, Gomes MB (2013) Metformin: an old but still the best treatment for type 2 diabetes. Diabetol Metab Syndr 5: 6 .

32. Vilsboll T (2009) Liraglutide: a new treatment for type 2 diabetes. Drugs Today (Barc) 45: 101-113.

33. Sun F, Yu K, Wu S, Zhang Y, Yang Z, et al. (2012) Cardiovascular safety and glycemic control of glucagon-like peptide-1 receptor agonists for type 2 diabetes mellitus: a pairwise and network meta-analysis. Diabetes Res Clin Pract 98: 386-395

34. Best JH, Hoogwerf BJ, Herman WH, Pelletier EM, Smith DB, et al. (2011) Risk of cardiovascular disease events in patients with type 2 diabetes prescribed the glucagon-like peptide 1 (GLP-1) receptor agonist exenatide twice daily or other glucose-lowering therapies: a retrospective analysis of the LifeLink database. Diabetes Care 34: 90-95.

35. Pottegård A, Christensen Rd, Houji A, Christiansen CB, Paulsen MS, et al. (2014) Primary non-adherence in general practice: a Danish register study. Eur J Clin Pharmacol 70: 757-763.

36. Marso SP, Poulter NR, Nissen SE, Nauck MA, Zinman B, et al. (2013) Design of the liraglutide effect and action in diabetes: evaluation of cardiovascular outcome results (LEADER) trial. Am Heart J 166: 823-830. 